\title{
Acute pseudogout of the foot following Parathyroidectomy: a case report
}

\author{
Sari Priesand ${ }^{*}$ (D), Jennifer Wyckoff, James Wrobel and Brian Schmidt
}

\begin{abstract}
Background: The current case report is the first in the literature to report the association between parathyroidectomy and an episode of probable pseudogout in the foot in a patient with diabetes mellitus.

Case presentation: The authors present a case of pseudogout of the left foot following a subtotal parathyroidectomy in an 80-year-old female with type 2 diabetes mellitus and primary hyperparathyroidism. Pseudogout, also known as calcium pyrophosphate dehydrate deposition disease, is an unusual metabolic sequela following parathyroidectomy. Pseudogout attacks have been noted in the immediate post-operative period following parathyroidectomy, but has rarely been identified in the foot. The diagnosis was challenging and there were multiple differential diagnoses that were entertained in this case.

Conclusions: This case illustrates the importance of reviewing the surgical history as there might be a link between the previous surgeries and current foot problems. This case also serves as a reminder of the importance of calcium and phosphate metabolism in podiatric health. Most specifically it demonstrates again the association of pseudogout and parathyroidectomy in a patient with diabetes mellitus. Although a rare occurrence, it is an important reminder that metabolic imbalances of calcium levels can manifest in any bone.
\end{abstract}

Keywords: Pseudogout, Parathyroidectomy, Calcium pyrophosphate dehydrate, Diabetes mellitus

\section{Background}

Calcium pyrophosphate dehydrate (CPPD) deposition disease is also known as chondrocalcinosis or pseudogout. The incidence of pseudogout in the United States is 275 cases per 100,000 people, roughly half the incidence of gout [1, 2]. Pseudogout occurs most often between the sixth and eighth decades of life and the male to female ratio is 1.5:1 [2, 3]. CPPD crystals are deposited in cartilage, synovial and capsular tissues as well as within tendons and ligaments. The most common areas of involvement are the knees, followed by the upper extremity joints and the metatarsophalangeal joint of the foot [1]. The crystals are radiopaque and appear as linear calcifications within soft tissues. Joint destruction, although uncommon, resembles osteoarthritis [1].

Although infrequent, pseudogout is a well-documented diagnostic clue of hyperparathyroidism or sequela

\footnotetext{
* Correspondence: sarig@med.umich.edu

Department of Internal Medicine, Division of Metabolism, Endocrinology, and

Diabetes, University of Michigan Hospital and Health Systems, Domino's

Farms Lobby G, Suite 1500, 24 Frank Lloyd Wright Drive, Ann Arbor, MI

48106, USA
}

following parathyroidectomy [4-10]. Hyperparathyroidism is known to increase the risk of CPPD, presumably due to long standing increased serum calcium [11]. Therefore, the exact mechanism by which a parathyroidectomy would precipitate acute CPPD is unclear. One commonly accepted theory is that following parathyroidectomy the relative fall in calcium levels may decrease the solubility of calcium pyrophosphate, precipitating the shedding of crystals out of the cartilage and into the synovial fluid, resulting in an inflammatory response [12]. Other possible explanations might be that the sudden decrease in the effect of parathyroid hormone (PTH) on the phosphate metabolism, either locally in cartilage or perhaps due to the drop in the renal excretion of phosphate, unbalances the equilibrium between the chondrocyte's production of inorganic pyrophosphate (iPP) and its hydrolysis to orthophosphate by tissue non-specific alkaline phosphatase. iPP is integral to the formation of CPPD crystals and therefore a relative, even transient, increase in iPP might be expected to increase the deposition of CPPD crystals [13]. Acute synovitis develops when the crystals produce an acute inflammatory response [1]. 
The diagnosis is challenging, especially in post-surgical patients in whom fever, leukocytosis, and pain may be attributed to other causes $[14,15]$. The differential diagnosis for this condition includes: gout $[16,17]$, hungry bone disease [18], Charcot neuroarthropathy, seronegative arthritis, osteoarthritis, rheumatoid arthritis, and cellulitis/septic arthritis.

Joint aspiration and microscopic identification of crystals are recommended to establish a definitive diagnosis. The microscopic demonstration of weakly positive birefringent crystals of varying shapes under polarized light (1 to $20 \mu \mathrm{m}$ in length) is diagnostic [1]. The recommended conservative treatment for pseudogout is pharmacologic management with NSAIDs, as well as the judicious use of intra-articular steroid injections $[1,19]$. Colchicine can be used [20], although it is relatively inconsistent in relieving symptoms; NSAIDs may be more helpful [1].

This case is unique as it is the first case in the literature to describe a case of pseudogout of the foot following parathyroidectomy in a patient with type 2 diabetes. Our case was complicated because our patient also had type 2 diabetes, making the diagnosis difficult. This emphasizes the importance of reviewing surgical history, as well as ruling out other possibilities in the differential diagnoses, in order to attribute the symptoms to a specific diagnosis and choose appropriate treatment.

\section{Case presentation}

Our patient is an 80-year-old female with past medical history of well-controlled type 2 diabetes mellitus for 37 years, chronic kidney disease stage 3 , monoclonal gammopathy of uncertain significance, chronic kidney disease stage 3 (stable for a decade), hypertension, obesity (BMI 39), GERD, hyperlipidemia, treated endometrial cancer, and primary hyperparathyroidism. She presented with an erythematous, painful, edematous left foot. Patient denied any history of smoking, alcohol, or illicit drug use. Patient denied any previous history of pedal complaints.

Patient had biochemical and radiographic evidence of primary hyperparathyroidism for 8 years and was being followed by both an endocrinologist and an endocrine surgeon. She had no clinical complaints related to her hypercalcemia, no history or ultrasonographic evidence for kidney stones, and excellent bone density. However, progressive hypercalcemia developed and prompted

Table 1 Patient's labs prior to parathyroidectomy

\begin{tabular}{ll}
\hline Lab test & Lab value \\
\hline Serum Calcium (Ca) $[8.6-10.3 \mathrm{mg} / \mathrm{dl}]$ & $10.4-11.9 \mathrm{mg} / \mathrm{dl}$ \\
Parathyroid Hormone (PTH) $[10-65 \mathrm{pg} / \mathrm{ml}]$ & $154 \mathrm{pg} / \mathrm{ml}$ \\
25 -hydroxyvitamin D [30-50 ng/ml] & $47 \mathrm{ng} / \mathrm{ml}$ \\
\hline
\end{tabular}

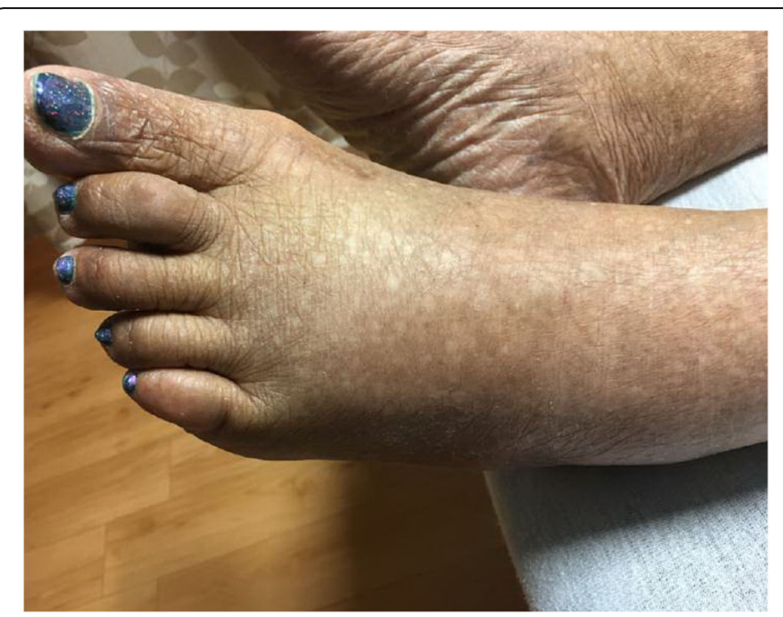

Fig. 1 Clinical image left foot

surgical intervention. Her pre-operative lab values are displayed in Table 1.

She underwent a subtotal parathyroidectomy of grossly enlarged right superior parathyroid sand left superior parathyroid glands. A morphologically normal right inferior parathyroid gland and a mildly enlarged left inferior parathyroid gland (that appeared to be largely replaced by adipose) were left in situ. PTH levels were

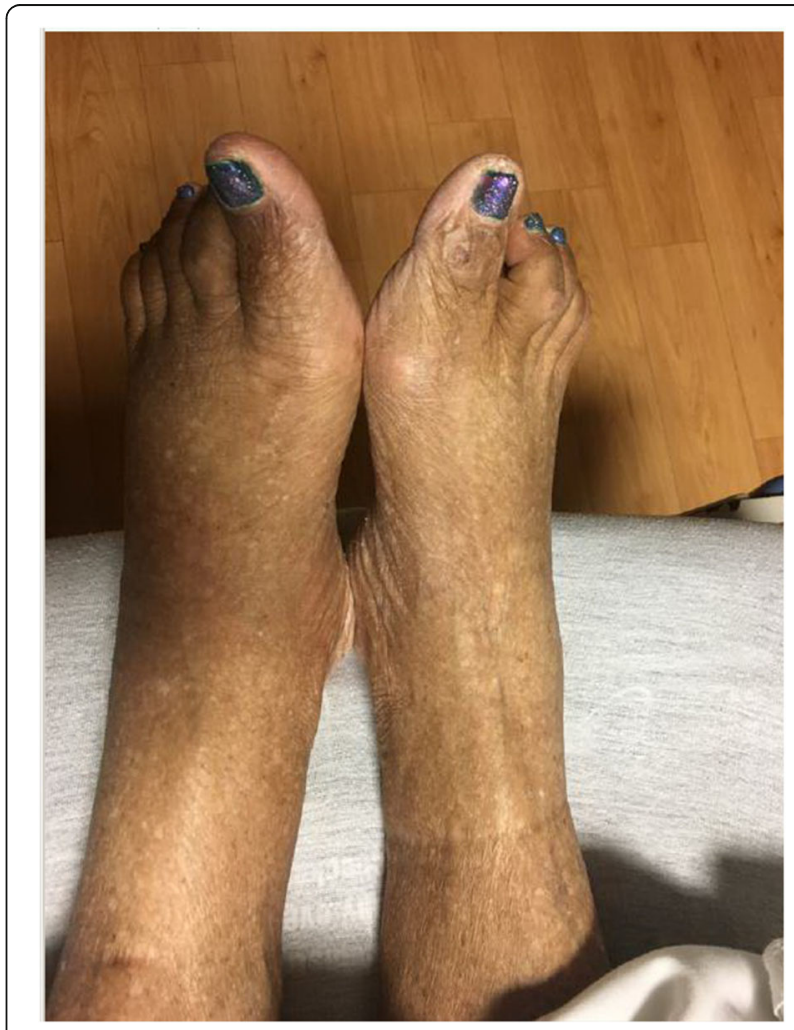

Fig. 2 Clinical image left foot vs. right foot 
Table 2 Patient's labs upon readmission status post parathyroidectomy

\begin{tabular}{ll}
\hline Lab test & Lab value \\
\hline WBC $[4.0-10.0 \mathrm{~K} / \mathrm{uL}]$ & $11.5 \mathrm{KuL}$ \\
BUN $[7-20 \mathrm{mg} / \mathrm{dl}]$ & $25 \mathrm{mg} / \mathrm{dl}$ \\
Cr $[0.5-1.1 \mathrm{mg} / \mathrm{dl}]$ & $1.42 \mathrm{mg} / \mathrm{dl}$ \\
ESR $[0-20 \mathrm{~mm} / \mathrm{h}]$ & $78 \mathrm{~mm} / \mathrm{h}$ \\
CRP $[\leq 3.0 \mathrm{mg} / \mathrm{dl}]$ & $12.6 \mathrm{mg} / \mathrm{dl}$ \\
Ca $[8.6-10.3 \mathrm{mg} / \mathrm{dl}]$ & $7.5 \mathrm{mg} / \mathrm{dl}$ \\
\hline
\end{tabular}

monitored throughout the procedure. An appropriate drop in PTH levels from $553 \mathrm{pg} / \mathrm{ml}$ (normal 12-75 pg/ml) baseline (right internal jugular) to $37 \mathrm{pg} / \mathrm{ml}$ was observed. Pathology revealed two enlarged, hyper-cellular glands. Her surgical procedure was without complication and she was discharged the day after surgery after an uncomplicated overnight stay. At discharge on post op day 1, PTH was $14 \mathrm{pg} / \mathrm{dl}$ and serum calcium was $8.3 \mathrm{mg} / \mathrm{dl}$.

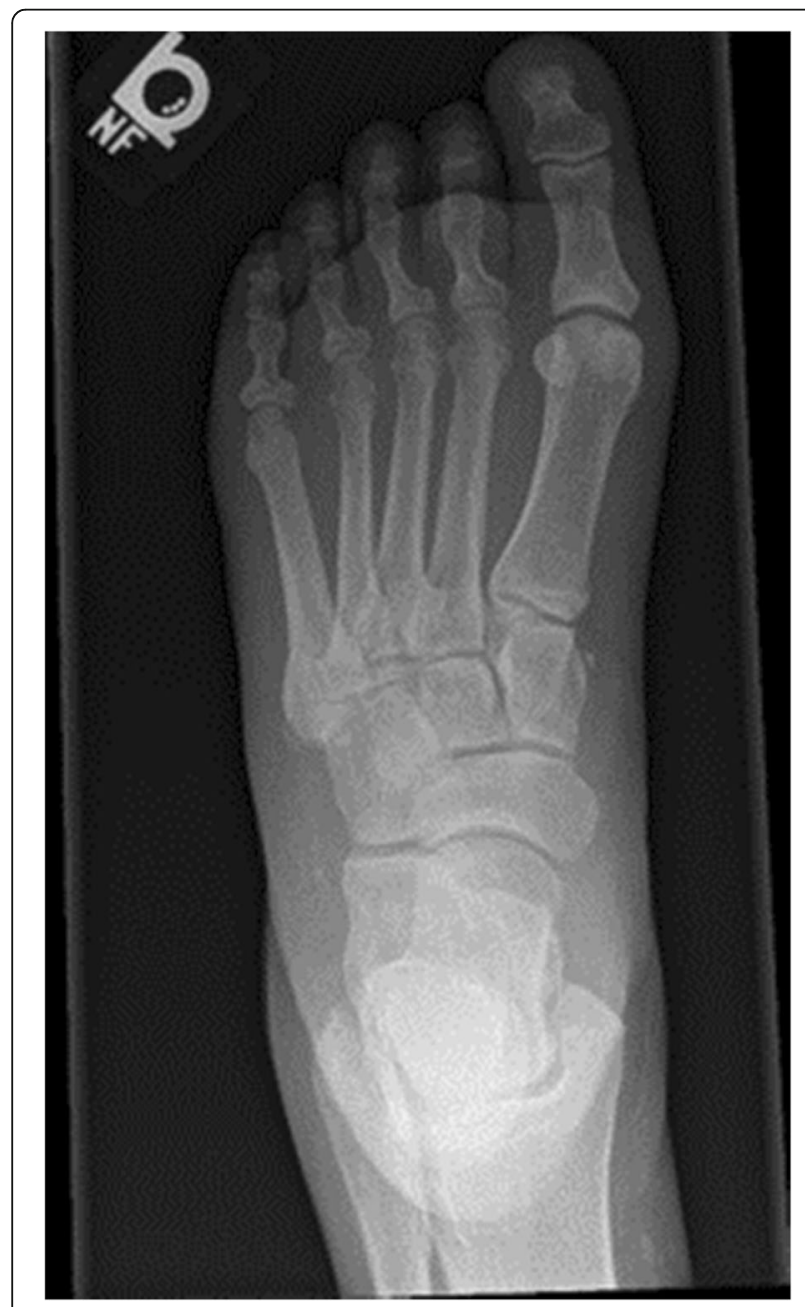

Fig. 3 X-ray left foot
Seven days post operatively, the patient developed left foot pain, swelling, warmth, and inability to bear weight (Figs. 1 and 2). She denied any precipitating trauma. She had no prior history of cellulitis or Charcot neuroarthropathy. She denied any numbness, burning, or tingling to her feet or any symptoms of peripheral neuropathy. She noted that the foot pain became progressively worse, particularly with standing. She otherwise denied any fever, chills, nausea, vomiting, chest pain, shortness of breath, abdominal pain, or diarrhea. Her initial labs on readmission are shown in Table 2.

There was radiographic evidence of soft tissue swelling with no findings concerning for osteomyelitis or Charcot neuroarthropathy (Figs. 3, 4, 5). She was admitted with the presumed of cellulitis of the left foot and was started on intravenous antibiotics (Zosyn and Vancomycin).

Three days following her admission, a podiatry consult was placed for evaluation of the left foot. Upon physical

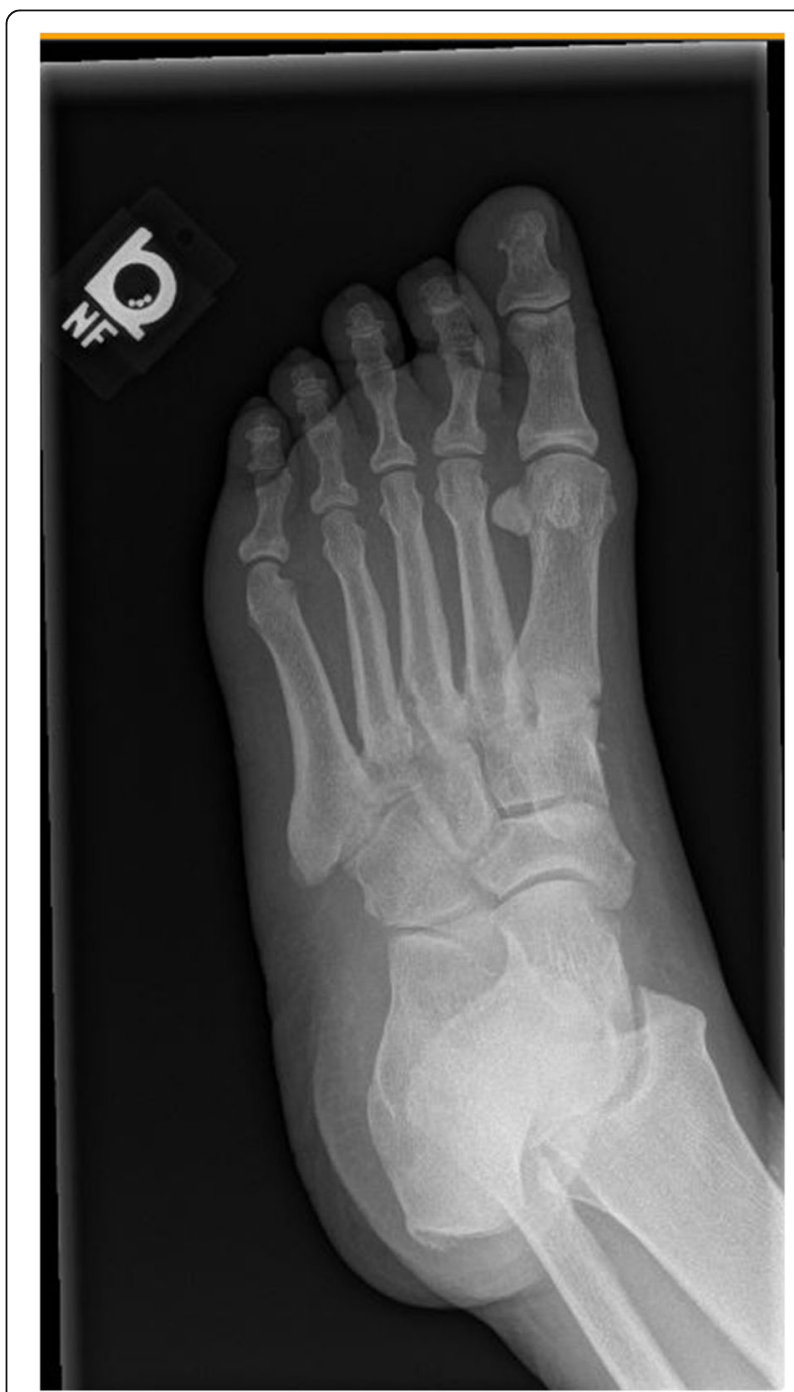

Fig. 4 X-ray left foot 


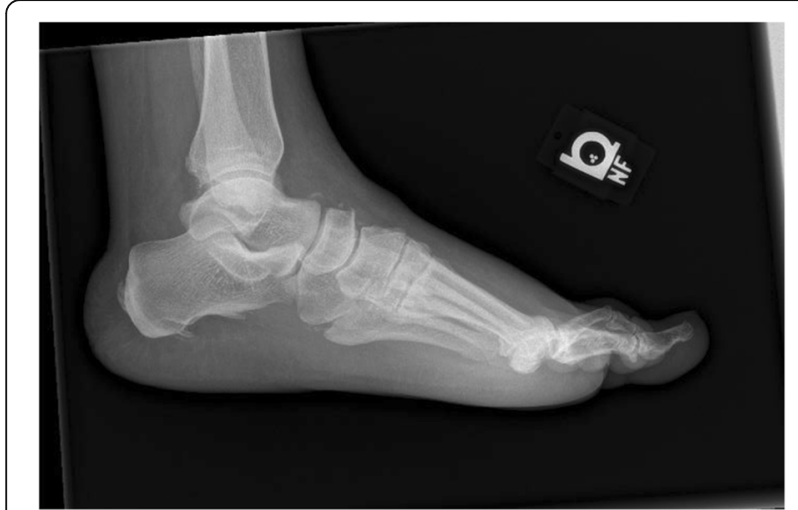

Fig. $\mathbf{5}$ X-ray left foot

exam patient had $2 / 4$ dorsalis pedis and posterior tibial pedal pulses bilaterally, capillary fill time was within normal limits and the left foot was warm to touch compared to the right foot. Protective sensation was evaluated via 5.07 monofilament, which revealed intact sensation to bilateral feet. Vibratory sensation was evaluated via timed clanging tuning fork and was measured to be $19 \mathrm{~s}$ at the right first metatarsophalangeal joint and $20 \mathrm{~s}$ at the left first metatarsophalangeal joint. Thus, the vibratory sensation was within normal limits to bilateral feet. On focused evaluation of the left foot, it appeared the foot was erythematous, warm and markedly swollen. There was no lymphadenopathy noted. There were no portals of entry visualized and no open wounds identified. There was pain on palpation specifically over the dorsal midfoot laterally. There was no crepitus noted on range of motion of the midtarsal or tarsometatarsal joint and no pain on range of motion of the individual metatarsals. No crepitus or fluctuance noted.

On initial assessment, the differential diagnosis of cellulitis versus Charcot neuroarthropathy was entertained.
The patient did not improve on broad spectrum antibiotics though her white blood cell count did normalize. She denied any systemic symptoms. The diagnosis of septic arthritis at this point seemed unlikely. Given lack of evidence of neuropathy, the diagnosis of Charcot neuroarthropathy seemed less likely. Dermal thermometry was performed bedside and the recordings (Fig. 6) were analyzed according to work of Armstrong et al. and Najafi et al. [21-23] The xrays were reviewed and there were no notable findings. Patient denied a history of gouty arthritis or pedal osteoarthritis. She reported that she did not have foot pain prior to admission. On further assessment and review of recent parathyroidectomy surgical history, podiatry recommended a CT scan (Fig. 7) for evaluation of the underlying osseous structures, an endocrinology evaluation due to patient's persistent hypocalcemia, and a rheumatology consult for evaluation for pseudogout. Patient was made non weight bearing in a CAM (controlled ankle movement) walker at this time as well.

Endocrinology was consulted for her hypocalcemia, and the recommendation for calcitriol $0.25 \mathrm{mcg}$ BID, continue calcium citrate-vitamin D3 supplement 2 tabs TID, and to continue vitamin D3 1000 units daily. They also recognized that hungry bone syndrome is another documented outcome following parathyroid gland removal, but deemed it unlikely in this case.

Upon evaluation by the rheumatology team, the diagnosis of a crystalline arthritis was evident. The recommendations included starting $0.6 \mathrm{mg}$ BID colchicine and then to continue with $0.6 \mathrm{mg}$ colchicine daily until first post discharge follow up, $10 \mathrm{mg}$ daily prednisone, and $100 \mathrm{mg}$ daily allopurinol after acute symptoms resolved. They recommended a uric acid level be drawn, which returned as $7.5 \mathrm{mg} / \mathrm{dL}$ (normal $2.2-6.0 \mathrm{mg} / \mathrm{dL}$ ). The CT scan revealed a constellation of findings suggesting

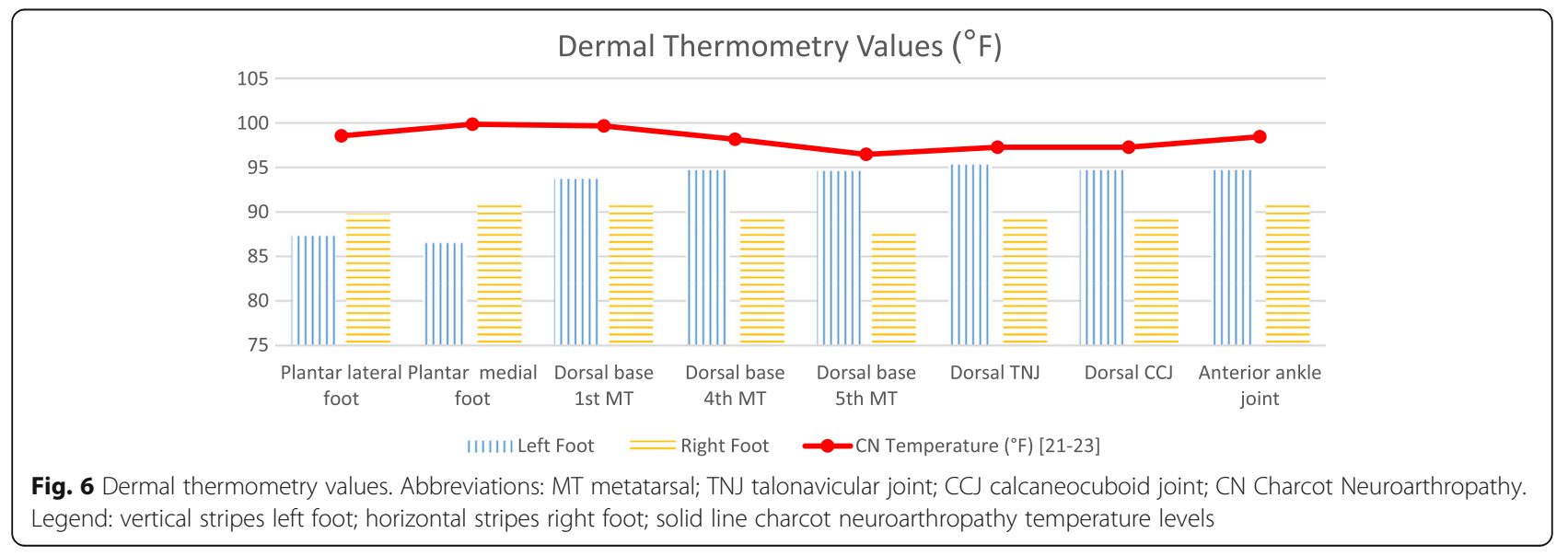




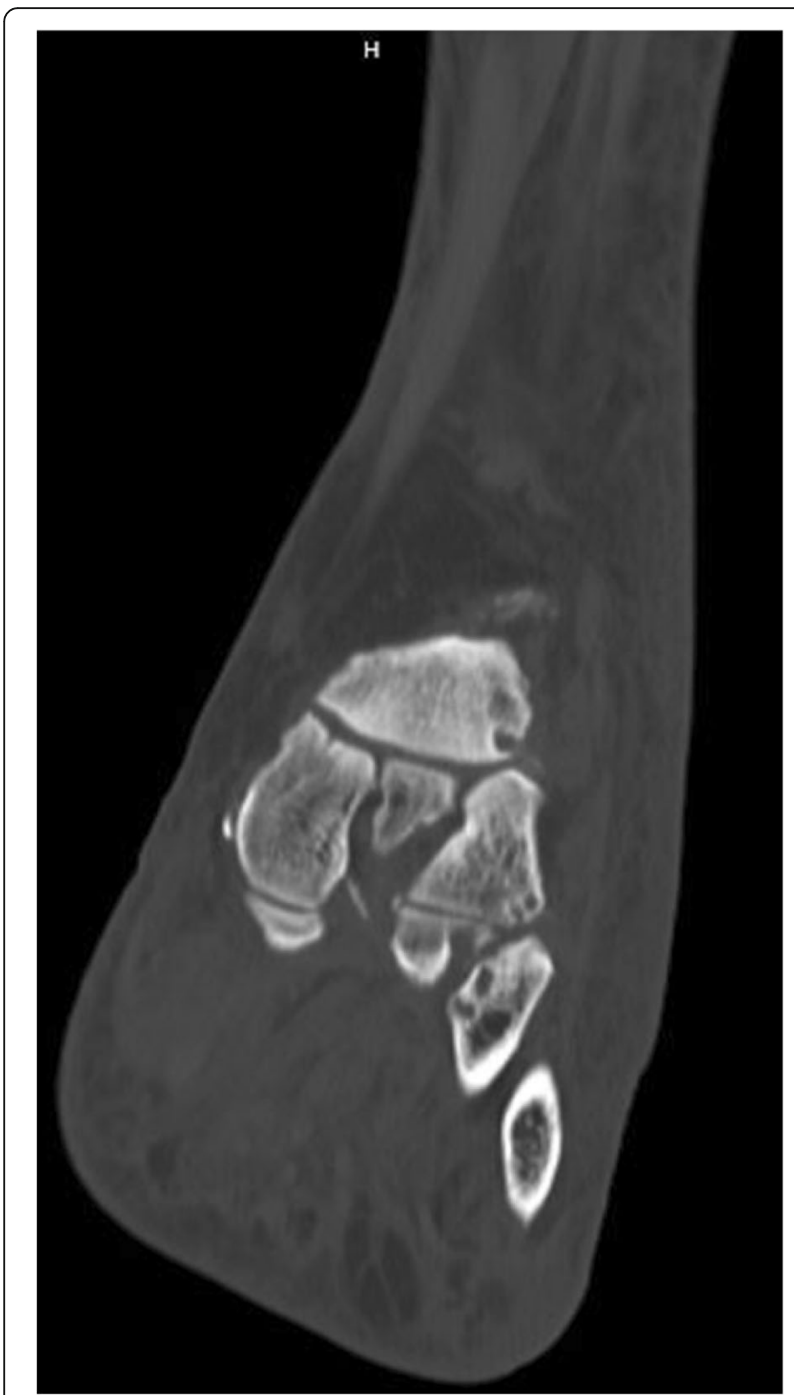

Fig. 7 CT scan left foot sequela of midfoot and ankle gout arthropathy. At this point the diagnosis of gout versus pseudogout was discussed and it seemed that due to patient's recent parathyroidectomy, pseudogout was more likely. NSAIDs were avoided in this case due to patient's compromised renal status. The rheumatology team also felt a joint aspiration at this point was not necessary, and attempting treatment with pharmacologic therapy first was most appropriate.

After beginning pharmacologic treatment as recommended by both the endocrinology and rheumatology teams, calcium levels began to slowly normalize (Fig. 8) and patient's left foot pain began to decrease. The patient's left foot edema reduced and the erythema resolved shortly thereafter. Patient at this point was transitioned to protected weight bearing of the left lower extremity in the CAM Walker with use of a walker and gait training with physical therapy.

\section{Discussion}

This case highlights the clinical presentation of pseudogout following parathyroid gland removal. The diagnosis of pseudogout is difficult due to the various diagnoses that mimic its clinical presentation. Specifically, when type 2 diabetes mellitus is present the additional diagnoses of septic joint/cellulitis and acute Charcot neuroarthropathy must be ruled out. $\mathrm{CN}$, when in Eichenholtz stage 0, is clinically difficult to diagnose, as there are no visible radiographic findings. Acute $\mathrm{CN}$ classically presents as an erythematous, edematous, warm, neuropathic/insensate foot. $\mathrm{CN}$, when in stages $1-3$, display various levels of radiographic joint fragmentation with subsequent remodeling and consolidation [24, 25]. Lomax et al. reviewed how the radiographic destruction seen in cases of CPPD can resemble $\mathrm{CN}$ [26]. In this case, the absence of peripheral neuropathy ruled out the possibility that $\mathrm{CN}$ was playing a role. The importance of reviewing the surgical history was vital in this case as it explained the patient's hypocalcemia in addition to the left foot symptoms.

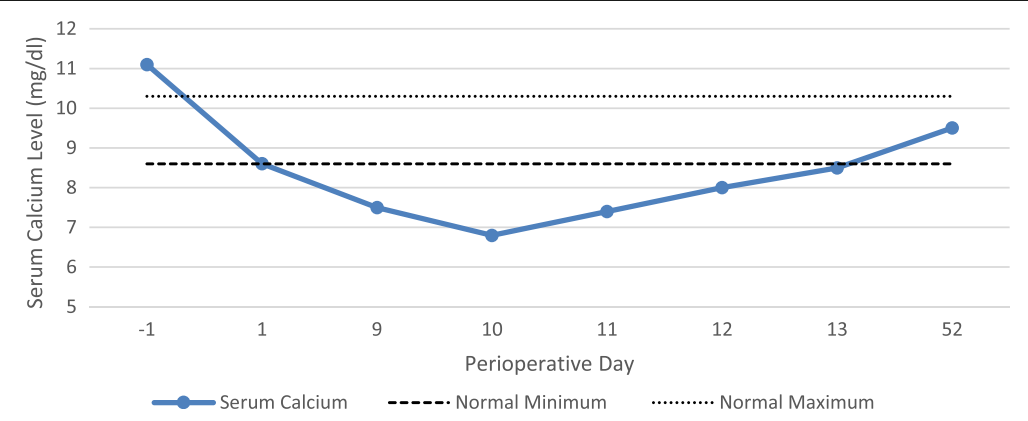

Fig. 8 Peri-operative serum calcium levels. Legend: solid line patient values; dashed points minimum normal values; dotted points maximum normal values 
In this case, performing a joint aspiration of the tarsometatarsal joint would have been definitive, as microscopic evaluation of the joint crystals would have differentiated between pseudogout and gout as well as definitively rule out septic joint. However, the risk of introducing bacteria into a non-septic joint was a concern and the rheumatology recommendation for pharmacologic therapy prior to joint aspiration was appreciated in this case. Zuber's study on knee joint aspirations and injections stated that the introduction of infection after injection is less than 1 in 10,000 procedures. He also addresses that a contraindication to aspiration is an inaccessible joint [27]. The tarsometatarsal joint anatomically is composed of multiple joint articulations including the tarsometatarsal, intertarsal, and proximal intermetatarsal joints. There are dorsal, plantar, and interosseous ligaments making the tarsometatarsal joints technically challenging to obtain an adequate synovial fluid sample [28]. Therefore, the rationale for administering pharmacologic medication prior to attempting synovial fluid analysis was deemed appropriate in this clinical setting. In terms of treatment, this patient responded quite well to colchicine and prednisone. NSAIDs in this case were avoided due to patient's history of renal compromise, but in an otherwise healthy patient NSAIDs could have been utilized.

Although not a factor in this particular case, as there were no significant destructive osseous changes postoperatively or bone symptoms pre-operatively, we wanted to briefly comment on hungry bone syndrome as it has not yet been addressed in the podiatric literature. Hungry bone syndrome occurs following parathyroidectomy, when sometimes profound hypocalcemia occurs due to the acute decline in PTH. As Brasier described in 1988, in the hyperparathyroid individual, PTH increased bone formation and resorption with a net efflux of calcium from the bone. Sudden withdrawal of PTH, as in parathyroidectomy, causes an imbalance between osteoblast-mediated bone formation and osteoclast-mediated bone resorption, leading to a marked net increase in bone uptake of calcium, phosphate, and magnesium [18]. Clinical features of this condition include hypocalcemia, hypophosphatemia, hypomagnesemia, and hyperkalemia. In Brasier's study, the incidence of hungry bone syndrome following parathyroidectomy was $12.6 \%$ [18].

\section{Conclusion}

This case illustrates the importance of reviewing the surgical history as there might be a link between the previous surgeries and current foot problems. This case also serves as a reminder of the importance of calcium and phosphate metabolism in podiatric health, as pseudogout, gout, and hungry bone syndrome were all entertained in this case. The clinical presentation of CPPD can be variable and is a great mimicker of other forms of arthritis as well as Charcot neuroarthropathy. Most specifically it demonstrates again the association of pseudogout and parathyroidectomy in a patient with diabetes mellitus and it describes it for the first time in the podiatric literature. Although a rare occurrence, it is an important reminder that metabolic imbalances of calcium levels can manifest in any bone.

\section{Abbreviations \\ BUN: Blood urea nitrogen; Ca: Calcium; CAM: Controlled ankle movement; CCJ: Calcaneocuboid joint; CN: Charcot neuroarthropathy; CPPD: Calcium pyrophosphate dehydrate; Cr: Creatinine; CRP: Creactive protein; ESR: Erythrocyte sedimentation rate; IPP: Inorganic pyrophosphate; MT: Metatarsal; PTH: Parathyroid hormone; TNJ: Talonavicular joint; WBC: White blood cell}

\section{Acknowledgements}

None.

Funding

None.

Availability of data and materials

Data sharing not applicable to this article as no datasets were generated or analyzed during the current study.

Authors' contributions

SP, JW, JW, and BS contributed edits of this manuscript and all read and approved the final manuscript.

Ethics approval and consent to participate

Written informed consent was obtained from the patient for publication of this case report and any accompanying images. A copy of the written consent is available for review by the Editor-in-Chief of this journal.

\section{Consent for publication}

Written informed consent was obtained from the patient for publication of this case report and any accompanying images. A copy of the written consent is available for review by the Editor-in-Chief of this journal.

\section{Competing interests}

The authors declare that they have no competing interests.

\section{Publisher's Note}

Springer Nature remains neutral with regard to jurisdictional claims in published maps and institutional affiliations.

Received: 7 September 2017 Accepted: 10 November 2017

Published online: 18 November 2017

References

1. Shurnas PA. Coughlin M. Surgery of the Foot and Ankle: Chapter 16 Arthritic Conditions of the Foot. 8th ed. 2007.

2. Beutler A, Schumacher HR Jr. Gout and 'pseudogout'. When are arthritic symptoms caused by crystal deposition? Postgrad Med. 1994;95(2):103-6. 109, 113-6 passim

3. Albin RK, Weil LS. Flexible implant arthroplasty of the great toe: an evaluation. J Am Podiatry Assoc. 1974;64(12):967-75.

4. Doshi J, Wheatley H. Pseudogout: an unusual and forgotten metabolic sequela of parathyroidectomy. Head Neck. 2008;30(12):1650-3.

5. Bilezikian JP, et al. Pseudogout after parathyroidectomy. Lancet. 1973:1(7801):445-6.

6. Nahir M, et al. Acute pseudogout attack following parathyroidectomy. Rheumatol Rehabil. 1978;17(1):6-7.

7. O'Duffy JD. Clinical studies of acute pseudogout attacks: comments on prevalence, predispositions, and treatment. Arthritis Rheum. 1976;19(Suppl 3):349-52. 
8. Kellett HA, MacLaren IF, Toft AD. Gout and pseudogout precipitated by parathyroidectomy. Scott Med J. 1982;27(3):250-1.

9. Glass JS, Grahame R. Chondrocalcinosis after parathyroidectomy. Ann Rheum Dis. 1976;35(6):521-5.

10. Kobayashi $\mathrm{S}$, et al. Two cases of acute pseudogout attack following parathyroidectomy. Endocrinol Jpn. 1991;38(3):309-14.

11. Pappu R, et al. Musculoskeletal manifestations of primary hyperparathyroidism. Clin Rheumatol. 2016;35(12):3081-7.

12. Geelhoed GW, Kelly TR. Pseudogout as a clue and complication in primary hyperparathyroidism. Surgery. 1989;106(6):1036-41. discussion 1041-2

13. Schlee S, Bollheimer LC, Bertsch T, et al. Crystal arthritides - gout and calcium pyrophosphate arthritis: part 1: epidemiology and pathophysiology. Z Gerontol Geriatr. 2017. doi:10.1007/s00391-017-1197-3. [Google Scholar].

14. White JC, Brandt FB, Geelhoed GW. Acute pseudogout following parathyroidectomy. Am Surg. 1988;54(8):506-9.

15. Masuda I, Ishikawa K. Clinical features of pseudogout attack. A survey of 50 cases. Clin Orthop Relat Res. 1988;229:173-81.

16. Loffler $\mathrm{C}$, et al. Distinguishing gouty arthritis from calcium pyrophosphate disease and other arthritides. J Rheumatol. 2015;42(3):513-20.

17. Martel W. Pseudogout vs. gout. Univ Mich Med Cent J. 1966;32(4):200-1.

18. Brasier AR, Nussbaum SR. Hungry bone syndrome: clinical and biochemical predictors of its occurrence after parathyroid surgery. Am J Med. 1988;84(4):654-60

19. Agarwal AK. Gout and pseudogout. Prim Care. 1993:20(4):839-55.

20. Alvarellos A, Spilberg I. Colchicine prophylaxis in pseudogout. J Rheumatol. 1986;13(4):804-5.

21. Armstrong DG, Lavery LA. Monitoring neuropathic ulcer healing with infrared dermal thermometry. J Foot Ankle Surg. 1996;35(4):335-8. discussion 372-3.

22. Armstrong $D G$, et al. Infrared dermal thermometry for the high-risk diabetic foot. Phys Ther. 1997;77(2):169-75. discussion 176-7

23. Najafi B, et al. Plantar temperature response to walking in diabetes with and without acute Charcot: the Charcot activity response test. J Aging Res. 2012;2012:140968

24. Eichenholtz S. Charcot joints. Springfield, IL, USA: Charles C.Thomas; 1966.

25. Rosenbaum AJ, DiPreta JA. Classifications in brief: Eichenholtz classification of Charcot arthropathy. Clin Orthop Relat Res. 2015;473(3):1168-71.

26. Lomax A, et al. Destructive pseudo-neuroarthropathy associated with calcium pyrophosphate deposition. Foot Ankle Int. 2015;36(4):383-90.

27. Zuber TJ. Knee joint aspiration and injection. Am Fam Physician. 2002;66(8):1497. -500, 1503-4, 1507

28. Weatherford BM, Anderson JG, Bohay DR. Management of Tarsometatarsal Joint Injuries. J Am Acad Orthop Surg. 2017;25(7):469-79.

\section{Submit your next manuscript to BioMed Central and we will help you at every step:}

- We accept pre-submission inquiries

- Our selector tool helps you to find the most relevant journal

- We provide round the clock customer support

- Convenient online submission

- Thorough peer review

- Inclusion in PubMed and all major indexing services

- Maximum visibility for your research

Submit your manuscript at www.biomedcentral.com/submit

) Biomed Central 